\title{
LA EDUCACIÓN SENTIMENTAL: Del paso de la ÉTICA de lÉvinas A LA PEDAGOGía DEL APRENDIZAJE
}

\author{
THE SENTIMENTAL EDUCACIÓN: THE TRANSITION FROM \\ LEVINAS ETHICS TO THE PEDAGOGY OF LEARNING
}

Por: Olga Elvira Acosta Amell*

*Administradora Educativa de la Universidad de San Buenaventura sede Bogotá; Magister en Ciencias de la Educación de la Universidad de San Buenaventura, sede Bogotá. Especialista en Gestión Educativa de la Universidad de Pamplona; Candidata a doctora en Humanidades, Humanismo y Persona con énfasis en Antropología Pedagógica de la Universida gica de la Universida de San Buenaventura, sede Bogotá. Rectora de la Institución Educativa Olga González Arraut. Directora de Investigación en la Maestría en Ciencias de la Educación de la Universida de San Buenaventura sede Cartagena. Direc tora de Investigación de la Maestría en Educación con énfasis en Gerencia de las Instituciones Educativas de la Universidad Tecnológica de Bolívar. Pertenica de Bolivar. vestigación Tendencias Actuales en educación y pedagogía TAEPE de la universidad San Buenaventura de Bogotá de la línea Antropologí Pedagógica Aśí mismo al grupo de investig al grupo de investigación interdisciplinario en Educación y Pedagogía GIEP. Universidac de San Buenaventura Cartagena.

\begin{abstract}
RESUMEN
Este artículo trata sobre la construcción de una pedagogía del aprendizaje a partir del planteamiento meta-ético expuesto en el texto Totalidad e infinito: Ensayo sobre la exterioridad de Emmanuel Lévinas. A la luz de conceptos tales como diálogo, enseñanza y formación, se establece una nueva forma de concebir la idea de la educación.
\end{abstract}

Palabras claves: Alteridad, diálogo, enseñanza, pedagogía del aprendizaje, meta-ética.

\begin{abstract}
This article is about the construction of a Pedagogy of Learning, using as a base the meta-ethical approach of the book Totality and Infinity: essay about exteriority written by Emmanuel Lévinas. Focused on concepts such as: dialogue, teaching and formation, we can propose a new way of conceive the idea of education.
\end{abstract}

Key words: Otherness, dialogue, teaching, Pedagogy of Learning,meta-ethics.

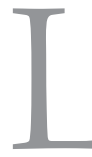

a importancia del pensamiento filosófico de Emmanuel Lévinas en nuestro proyecto argumentativo subyace en su vinculación directa

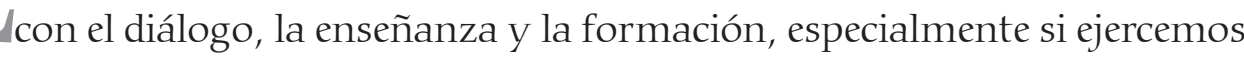
una lectura atenta de Totalidad e infinito: Ensayo sobre la exterioridad (2002), en donde no hay un solo argumento que deje de transmitir los procesos dialógicos entre las personas y sus consecuencias positivas en el ser.

Ahora, abordar estos tres conceptos (diálogo, enseñanza y formación) desde la obra del filósofo lituano, implica entender el trayecto que va de la interioridad del ser a la exterioridad del mismo, y con ello, el fenómeno moral de comprender al otro. En su ensayo sobre la exterioridad Lévinas maneja todo un discurso ético y político a partir de la alteridad, donde existe una permanente relación entre un YO interesado en trascender hacia el OTRO y su comportamiento en la sociedad, la familia, la escuela y la calle. Esta correlación YO-OTRO se funda en un nexo de responsabilidad y amor

Palubra No. 16. Agosto de 2016 
en el que no hay lugar a la neutralidad ni a la indiferencia por los demás. En este sentido, las guerras son el resultado de una mala interpretación del otro; los campos de concentración construidos por la Alemania nazi fueron el producto de una filosofía del poder que desarrolló falencias frente a los problemas de no saber entender a los otros.

Pero para entrar de lleno en el paso de la interioridad a la exterioridad convendría aclarar primero las categorías que intervienen en este proceso y sus características principales. Después de todo, la finalidad de este artículo es demostrar que en el trayecto que parte del YO hacia el OTRO es donde acontece, en gran medida, la formación ético-antropológica del sujeto que servirá para determinar las bases de una pedagogía del aprendizaje.

Entre los elementos esenciales del discurso de Lévinas, el primero es el YO, definido como el en sí de la persona que va hacia el OTRO. Este YO viene condicionado por el entorno social y la cultura en la que se desenvuelve, además de sufrir cambios a partir de la visión que obtiene de las personas a su alrededor. El YO se transforma a su vez en el MISMO, el cual busca la posibilidad de conocer y de juzgar al OTRO tomando como base su propia individualidad. Es el MISMO el que dinamiza la verdadera esencia que rige las relaciones de alteridad, sin que por eso digamos que actúa como protagonista, pues si en algo ha sido claro Lévinas es en establecer un equilibrio participativo entre sus categorías.

Por su parte, el OTRO es un ser físico y, sobre todo, metafísico, que se va constituyendo en la revelación de su rostro, suceso denominado epifanía. El OTRO expresa en su rostro la exterioridad a la que accede el YO, exterioridad que es infinita debido a su carácter metafísico. Es significativo recordar que lo infinito en el pensamiento de Lévinas supone lo perfecto, el bien supremo, y en últimas encierra la idea de Dios. Luego entonces es fundamental ver que Lévinas no solo legitima al OTRO como parte de un gran compromiso éticoantropológico, sino que también le otorga una especie de valor metafísico que justifica la presencia de Dios en el prójimo. Ahora, si la totalidad del OTRO es infinita, no se podría hablar de un Tú, puesto que esa sería una designación finita que ratifica un dominio y una calificación cerrada. Por eso cuando se escoge el concepto de OTRO, se hace con base a una lógica ética y no racional, en la cual el YO nunca va a poder conocer en su totalidad al OTRO porque es tan diferente a él, de tal forma que apenas si le será posible acceder al rostro. El único puente que garantiza dicho acceso a la exterioridad del OTRO es el lenguaje, el cual fluye activamente entre los seres, no solamente como 
${ }^{1}$ En Lucas 10:27 está escrito: "Amarás al Señor Dios tuyo de todo corazón, y con toda su alma, y con todas tus fuerzas, y con toda tu mente: $y$ al prójimo como a ti mismo": mientras que en Mateo 22. 34 40 está escrito: "Pero a los Phariséos, informados de que había tapado la boca a los Sadducéos, se mancomunaron: y uno de ellos, doctor de la Ley, le preguntó para tentar-

le: Maestro icúl es el mandamier mandarionto principal Jesús: amarás al Seño Dios tuyo de todo corazón, y con toda tu alma y con toda tu mente. Este es el máximo y primer mandamiento El segundo es semejante a ester es: amarás a tu prójimo como a ti mismo. En estos dos mandamientos está cifrada la Ley de los profetas". Curiosamente se advierte que en los mensajes de los evangelios hay igualentre Dios y el prójimo, puntualizando en el hecho de que amar a uno equivale a amar al otro, muy parecido a lo que postula Lévinas cuando le otorga un valor metaf́sico a OTRO tansico al OTRO relaciona con la infinitud de Dios, situándolo como parte de él. palabra oral y escrita, sino como fenómeno comunicativo en general donde las caricias, los gestos, los abrazos y la proxémica también contribuyen al acercamiento entre el YO y el OTRO.

El rostro, considerado como la revelación del OTRO, despierta el deseo del "Yo para el Otro", dicho deseo consiste en el reconocimiento compasivo que ejerce el YO sobre la exterioridad del OTRO, llegando al punto de imaginarlo como un YO poseedor de la misma dignidad y los mismos derechos. En Mr. Vértigo (Auster, 1995), la novela del escritor estadounidense Paul Auster, un personaje expresa lo siguiente: "si miras la cara de alguien durante el tiempo suficiente, acabarás por sentir que te estás mirando a ti mismo" (p.42). Ese es precisamente el mensaje de Lévinas, encontrar un espejo en los demás y darnos cuenta de que en ellos yacen las mismas convicciones que nos llevan a creer que somos dignos de ser respetados y comprendidos en nuestra diferencia, es decir: debemos mirar al otro y defenderlo como quien pretende defender su propia individualidad. Este es un postulado que se corresponde con algunas ideas de la filosofía cristiana, con aquello de amarás a tu prójimo como a ti mismo ${ }^{1}$. Del OTRO al YO hay una conexión de amor y compasión, sustentada en el hecho de que cuando el YO trasciende hacia el OTRO logra comprender su feminidad, que no es otra cosa que la fragilidad que se descubre en el OTRO.

Si dispusiéramos de una gráfica para mostrar en plena acción todas las categorías de Lévinas sobre el trayecto que se recorre del YO al OTRO, obtendríamos la siguiente representación:

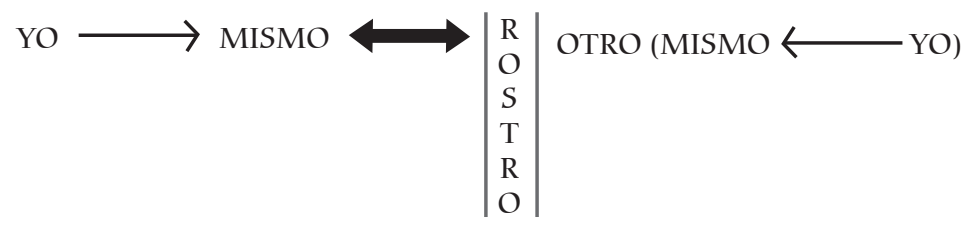

Allí el YO es recíproco con la exterioridad del OTRO concretada en su rostro, sin que por eso el OTRO deje de ser otro YO que busque acceder a la exterioridad de su prójimo.

Al llegar a este punto, en el que hemos explicado el proceso de la interioridad a la exterioridad y lo hemos juzgado como un proceso cardinal para el fenómeno moral de la comprensión del otro, es posible comenzar a discutir los conceptos de diálogo y enseñanza en el pensamiento filosófico de Lévinas y su incidencia en la formación ético-antropológica del sujeto. 


\section{Diálogo y enseñanza}

Para Lévinas, el diálogo es una relación de generosidad construida por dos o más visiones heterogéneas que se encuentran y que han sabido trascender la cerrada esfera de su individualidad. Este modo de concebir el diálogo sugiere una ética universal, en el entendido de que la universalidad de la razón surge de la superación de la subjetividad encerrada en sí misma, algo que evidentemente se consigue a través de las relaciones de alteridad. En consecuencia, el fundamento de la objetividad consiste en un enfrentamiento pacífico de múltiples consideraciones que emanan de cada uno de los seres humanos. Se trata, pues, de una construcción dialógica y dialéctica, más que de una visión en común.

En ese sentido, si 1, 2 y 3 piensan de forma A, B y C, respectivamente, la situación ideal que se busca no es un pensamiento $\mathrm{D}$ en donde se homogeneicen los sujetos, sino un ambiente propicio para la convivencia de A, B y C. Así, la primera conclusión a la que llegamos es que la propuesta ética de la filosofía levinasiana es una apología a la diversidad, una ardua defensa a los atributos individuales y su necesidad de coexistir en la absoluta paz que se genera a partir de la comprensión.

En el diálogo se recurre siempre a la relación "cara a cara", en ella se reconoce el rostro del OTRO y su dignidad como ser humano. Ahí, el YO no solo mantiene una actitud activa y se lanza hacia la exterioridad del OTRO, sino que también es sujeto pasivo del OTRO cuando este lleva toda su singularidad hasta el exterior, completando el ciclo de una verdadera alteridad que ciertamente no se limita a la actividad de un solo sujeto cognoscente.

Como hemos venido diciendo, la totalidad del ser es infinita y, por lo tanto, el YO nunca va a poder abarcar completamente al OTRO, solamente será capaz de conocer su rostro, que es la fenomenalización parcial del noúmeno. Lo que concretiza a la infinitud entre un ser y otro es el lenguaje, el cual funciona como un soporte material y temporal para los conceptos y las sustancias que se articulan en los individuos. El diálogo es lenguaje, su principal cualidad reside en su no-violencia, muy a pesar de que dentro de él se libre un choque de puntos de vista y de apreciaciones propias de la pluralidad de pensamientos.

La relación "cara a cara" (perteneciente más que todo a los seres humanos) rompe con la totalidad y la hace trascendente, suprime la coacción y el 
poder por el discurso mutuo, la homogeneidad por la diversidad, la libertad individual por la libertad colectiva y el subjetivismo ególatra por la alteridad, revelándonos una verdad en constante locomoción, muy parecida a la idea heracliteana del devenir. La verdad, a fin de cuentas, acaba siendo expresión en movimiento.

Todo este proceso dialógico entre las personas tiene importantísimas implicaciones en la enseñanza, especialmente si se le emparenta con la pedagogía. Según Rafael Flórez Ochoa en su libro Pedagogía y verdad (Ochoa, 1989), la pedagogía es un proceso de mediación entre el horizonte del aprendiz y el horizonte del saber; en esta mediación el pedagogo, es decir, el maestro, es quien hace apertura de los horizontes, cumpliendo una función central en los procesos de desarrollo cognitivo de los estudiantes, generándoles, a su vez, un impacto social, emocional y espiritual. Lévinas, por su parte, afirma que pese a que la exterioridad de los demás influye en mi desarrollo como individuo, las relaciones entre los seres se dan en la diferencia y no requieren mediación alguna. Esto significa que el encuentro con el otro se traduce en el recibimiento del otro, no para dominarlo ni sobrepasarlo, sino para aceptarlo como un ser independiente, pues la filosofía de Lévina tiene como objetivos la proximidad y la diversidad, no el poder ni la lejanía. Al vincular estos dos enunciados sobre las interacciones sociales, surge la gran pregunta de este artículo: cexiste la posibilidad de que esta meta-ética de Lévinas pueda ser considerada como una especie de meta-pedagogía? Nosotras pensamos que sí, quizás porque es el mismo filósofo quien parece sugerir esta conexión:

Abordar el Otro en el discurso, es recibir su expresión en la que desborda en todo momento la idea que implicaría un pensamiento. Es pues, recibir del Otro más allá de la capacidad del Yo; lo que significa exactamente: tener la idea de lo infinito. Pero eso significa también ser enseñado. La relación con Otro o el Discurso, es una relación no-alérgica, una relación ética, pero ese discurso recibido es una enseñanza. Pero la enseñanza no se convierte en la mayéutica. Viene del exterior y me trae más de lo que contengo. (Lévinas, 2002, p.75)

La educación, partiendo desde el pensamiento levinasiano, se debería entender como una relación ética con el otro, la educación es igualmente un "cara a cara" con los demás en el que mucho antes de asimilar a los otros a mi mismidad debo dejarlos ser, recibirlos con una hospitalidad incondicionada, transformando la apertura a la exterioridad del otro en un requisito previo y 
necesario a todo proceso pedagógico y educativo. Entonces la conexión entre la ética y la pedagogía se vuelve evidente, aunque hay un aspecto importante que debe quedar claro: Lévinas no construye una meta-pedagogía, sino que por simple coherencia toda meta-pedagogía requiere en primera instancia de una meta-ética, y esta sí es propuesta por Lévinas, de ahí que podamos enlazar los dos conceptos.

Siguiendo esta lógica, la educación no tiene como eje la enseñanza sino el aprendizaje $e^{2}$. Uno debe tanto aprehender al otro como aprender del otro. El aprendizaje se vuelve una relación interpersonal que implica salir de la interioridad de los saberes y de las estructuras preconcebidas. Reconocer la educación tomando como fundamento la acepción clásica de la enseñanza sería volver a la posición donde al otro se le asimila con violencia y no se le deja ser, donde se hacen enfáticas las jerarquías y la subordinación.

Esta problemática fue también abordada por el filósofo colombiano Estanislao Zuleta de forma muy persistente, llegando a concluir que una educación sin comprensión era una acción intimidadora del pensamiento. Así, en una de sus tantas entrevistas académicas terminó declarando:

La educación, tal como ella existe en la actualidad, reprime el pensamiento, transmite datos, conocimientos, saberes y resultados de procesos que otros pensaron, pero no enseña ni permite pensar. A ello se debe que el estudiante adquiere un respeto por el maestro y la educación que procede simplemente de la intimidación.

[...] Lo que se enseña no tiene muchas veces relación alguna con el pensamiento del estudiante, en otros términos, no se lo respeta, ni se lo reconoce como un pensador y el niño es un pensador.(Zuleta, 2009, p.19).

Los planteamientos de Lévinas como los de Zuleta nos colocan a reflexionar sobre la posibilidad de una educación que no gire en torno a la imposición de conocimientos sino en relación a presencias vivas que se comunican y se reconocen. Con estas ideas, lo que en realidad se enseñaría sería la presencia del otro. Aquí la lengua y el lenguaje actuarían como los vasos comunicantes de la alteridad, como las vías de doble calzada de la correspondencia entre las personas. La figura del maestro no sería, por lo tanto, la de un simple emisor de determinado contenido, sino que haría parte de esa creación de sentido

${ }^{2} \mathrm{Al}$ principio del escrito y en el subtítulo que encabeza esta parte del artículo hemos postulado el concepto de "eñ concepto de "eñananza no el de aprendizaj dentro del marco conceptual vinculado al pensamiento filosófico de Emmanuel Lévinas esto se debe a que el concepto de "enseñanz" al que hacemos re ferencia se compren ferencia se comprende como la enseñanza del
aprendizaje. 
que es el aprendizaje en sí mismo. Así, el maestro integraría la construcción de un sentido que tendría como fundamento el reconocimiento del otro.

Hace ya numerosas décadas que Gustav Flaubert escribió y publicó su novela La educación sentimental (Flaubert \& Luna, 2004) Sin embargo, aunque el tiempo haya ejercido su hegemonía de épocas y de olvidos, aquel título se ha seguido usando para definir y metaforizar muchas visiones del mundo. La visión del mundo de Lévinas, su propuesta ética, no es más que las pautas de una educación sentimental. Si no, cómo explicar que el recorrido de un ser a otro esté dado por un deseo infinito y no total, cómo explicar que la sociedad se convierta en un gigantesco "Nosotros" donde la máxima premisa social involucre un profundo compromiso moral con los demás. Cómo explicar, desde luego, que la educación termine siendo más un propósito emocional que un fenómeno intelectual, si se entiende por intelectual el conocimiento científico impuesto por los paradigmas de la academia. La clave de la pedagogía perfecta no reside en el compendio de saberes del maestro ni en la capacidad mental del estudiante, sino en el tipo de educación sentimental que se posea al momento de interactuar con nuestros semejantes.

\section{BIBLIOGRAFÍA}

Auster, P. (1995). Mr. Vertigo. Penguin. Retrieved from https://books.google.com.mx/books?hl= es\&lr $=\& \mathrm{id}=5 \mathrm{IJKKTFX33EC \& oi}=\mathrm{fnd} \& \mathrm{pg}=\mathrm{PT} 5 \& \mathrm{dq}=$ Auster,$++\mathrm{Mr} .+\mathrm{V} \% \mathrm{C} 3 \%$ A9rtigo.$+\&$ ots $=\mathrm{yPU}$ EvYQ2DX\&sig =ou4q4g5vTzs6OFh-gq9tqzYNPmk

Flaubert, G. \& Luna, R. (2004). La educación sentimental. LD Books. Retrieved from https:// books.google.com.mx/lr =\&id=lc2Sfe5KyJQC\&oi=fnd\&pg $=$ PA7 $\& d q=$ Gustav + Flaubert + la + edu cacion + sentimental\&ots $=$ QHJY10K716\&sig $=$ hbw1H6FlNggcZcAjOx2rS2Rqs7U

Lévinas, E. T. (2002). Infinito. Salamanca: Ediciones Sígueme. Retrieved from http://www. sigueme.es/docs/libros/totalidad-e-infinito-2012.pdf

Ochoa, R. F. (1989). Pedagogía y verdad (Vol. 4). Ediciones Secretaría de Educación y Cultura.

Zuleta, E. (2009). Educación y democracia, un campo de combate. Bogotá. Un Hombre Nuevo Editores. 

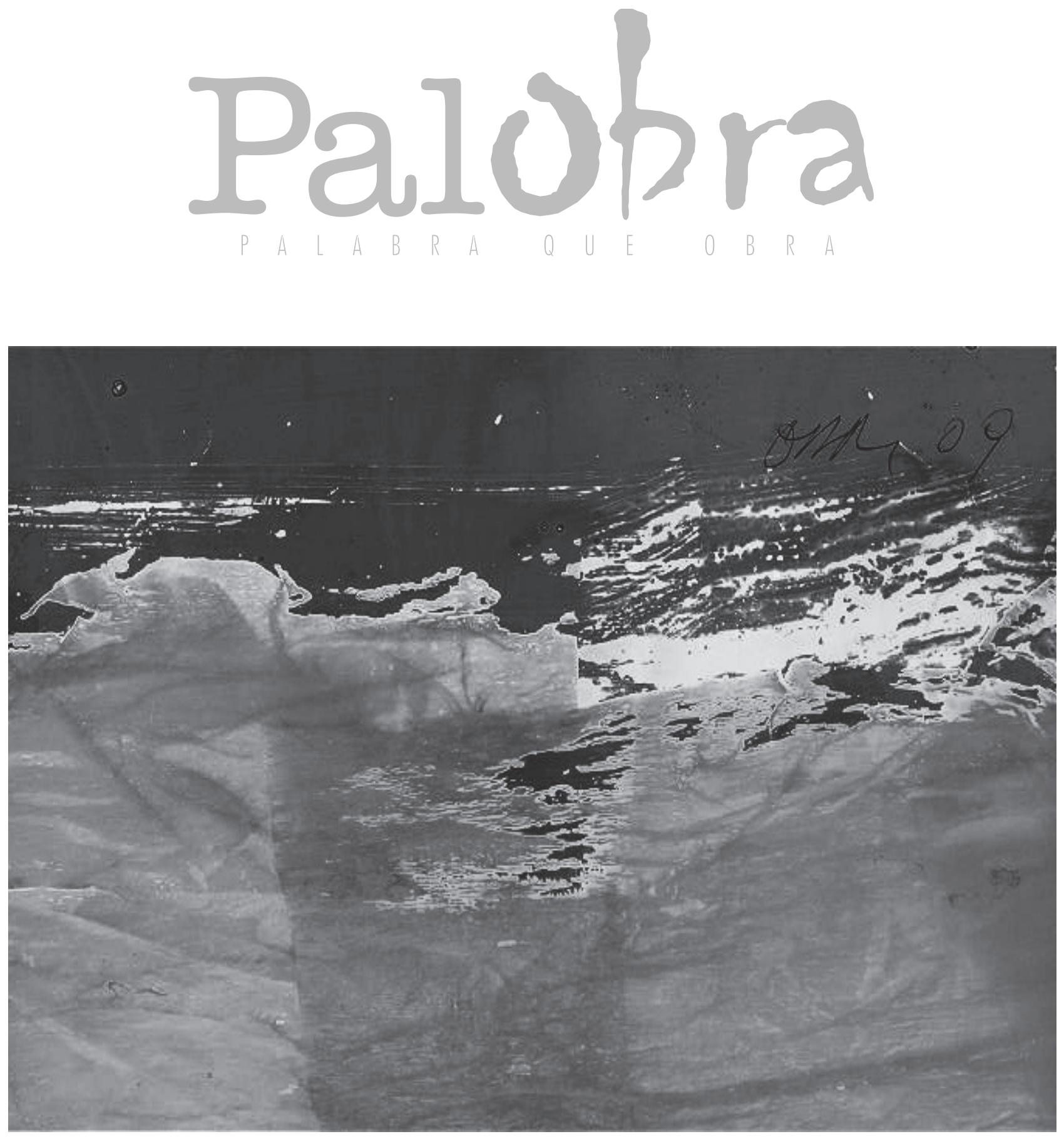

Paisajes a color. 2009. Impresión fotográfica sobre papel emulsionado. 